\title{
Confiance, violence et politique: quelques repères historiques et théoriques
}

\section{Confiança, violência e política: algumas referências históricas e teóricas}

Jacques Rhéaume - Ph. D. en Sociologie, est professeur émérite à l'UQAM. Ses champs d'intérêt sont la psychosociologie des groupes et des organisations, la santé mentale au travail, l'action communautaire et les approches d'histoires de vie. E-mail: rheaume.jacques@uqam.ca.

\section{Résumé}

Le phénomène social de la violence plonge ses racines dans les relations étroites entre les individus et le social, entre la dynamique psychique et les rapports sociaux. L'établissement d'une attitude de confiance envers l'autre, mais aussi par rapport aux institutions sociales et politiques est un facteur déterminant. Il convient d'abord de bien définir la notion de violence et ses diverses formes pour ensuite dégager le contexte sociohistorique de ce phénomène à partir d'auteurs qui ont tenté d'en expliquer les fondements, tant en psychologie (Freud par exemple) qu'en philosophie ou en sociologie (Hobbes, Rousseau, Enriquez). Les travaux de Jan Philipp Reemtsma nous offrent à cet égard un guide important pour situer la crise actuelle de confiance dans nos sociétés contemporaines et la montée correspondante de formes nouvelles de violence. Nous concluons en appelant au rôle majeur des chercheurs et intervenants en sciences humaines dans la reconstruction du lien social fondé sur la confiance.

\section{Mots clés}

Violence. Lien social. Confiance. Historicité. Psychosociologie.

\section{Resumo}

O fenômeno social da violência tem suas raízes das relações estreitas entre os indivíduos e a sociedade, entre a dinâmica psíquica e as relações sociais. O estabelecimento de uma atitude de confiança em relação ao outro, mas também em relação às instituições sociais e políticas, é um fator determinante. É necessário, inicialmente, definir bem a noção de violência e suas diversas formas para, em seguida, demonstrar o contexto sóciohistórico desse fenômeno a partir dos autores que tentaram explicar seus fundamentos, seja da psicologia (Freud, por exemplo), ou da filosofia e da sociologia (Hobbes, Rousseau, Enriquez). Os trabalhos de Jan Philipp Reemtsma nos oferecem, sobre o assunto, um guia importante para situar a crise atual de confiança nas sociedades contemporâneas e o avanço correspondente de novas formas de violência. Nós concluímos chamando atenção para $\mathrm{O}$ papel principal de pesquisadores e daqueles que fazem a intervenção em ciências humanas, de reconstrução do vínculo social fundado na confiança.

\section{Palavra-chave}

Violência social. Confiança. Historicidade. Psicosociologia. 


\section{INTRODUCTION}

La violence dans nos sociétés, du Nord au Sud, de l'Ouest à l'Est est d'une présence récurrente et troublante. Meurtres, attentats terroristes, agressions physiques mais aussi harcèlements, exploitations, racisme et discrimination. Et la violence se déploie aussi bien à l'égard d'individus que rapport à des collectivités importantes. Elle s'exerce certes suivant des degrés divers, plus ou moins destructrice, mais dans tous les cas elle représente le contraire d'une reconnaissance de l'autre dans le débat, la discussion, le partage du pouvoir, le dialogue, le lien social. Ce lien social dont la condition fondamentale est la confiance en l'autre, et la confiance réciproque.

Nous présentons quelques réflexions sur le phénomène de la violence dans ses multiples manifestations dans les rapports humaines et dans les sociétés, en donnant quelques repères historiques et théoriques pour tenter d'en cerner les fondements sociaux. Après avoir esquissé une définition et une phénoménologie de la violence, nous examinons plus spécifiquement les liens entre la violence et le pouvoir politique, les rapports étroits entre la confiance des membres d'une société à l'égard des institutions et la violence qui résulte d'une perte de cette confiance et les formes répressives qu'elle entraîne. C'est la base même de la construction du lien social que certains auteurs comme JeanJacques Rousseau et d'autres appellent le «contrat social» qui est ainsi mise en cause. Mais c'est aussi tout le développement du grand projet démocratique de la modernité qui est interpellé. Des éclairages issus de la philosophie, de la psychologie et de la sociologie viennent compléter ce questionnement. Enfin, il important aussi d'indiquer quel rôle peuvent jouer les chercheurs et praticiens des sciences humaines et sociales dans le contexte actuel de nos sociétés, d'éclairer les conditions et limites de leur «pouvoir d'agin».

\section{LES MOTS POUR LE DIRE: VIOLENCE, AGRESSION, CONFLIT, POUVOIR}

Le mot violence (violência) désigne l'acte d'exercer une force. Son origine étymologique est latine, de $V$ is, force, puissance. Il est à retenir que dans les usages courants les mots violenter, faire violence, violer, violence sont le plus souvent associés à l'usage abusif de la force, la force physique d'abord, puis psychologique (violence symbolique). A noter aussi, que la violence est à rapprocher d'un terme qui évoque aussi force et énergie, la virilité, de Vir, homme masculin en latin. 
L'agression (agressão), du latin aggressio (ad-gradior), désigne aller vers (d'où pro-gression et ré-gression), entreprendre, mais aussi, se porter à l'attaque, ce qui est le sens le plus courant... Elle désigne d'abord un mouvement vers autrui, un processus, et cette notion implique une force pour faire ce mouvement, cette attaque qui peut prendre diverses formes violentes.

La discussion de cette notion, dans un vieux manuel de psychologie (JOHNSON, 1972), montre que ce terme est polysémique et résiste à toute définition conceptuelle rigoureuse, compte tenu de la grande diversité des modes d'expression et des sources explicatives de l'agressivité humaine: biologique, développementale, sociale, éducative... Par exemple, et en partage avec la vie animale, l'agressivité se déploie dans la défense du territoire (et sa conquête); de l'espace personnel et privé; de la quête de la nourriture; elle se manifeste dans l'établissement des ordres de dominance et de statut (pecking order ou hiérarchie du becquetage chez les oiseaux); elle découle souvent des effets de promiscuité et de densité populationnelle. Une remarque de l'auteur est à souligner: l'agression animale, même mortelle, est rarement violente, au sens d'un usage abusif de la force, contrairement à ce qui se passe chez l'homme.

La notion de conflit (conflito) est déjà plus complexe. Elle évoque aussi l'usage de la force (fligere en latin veut dire heurter, s'entre-choquer) mais, elle implique aussi con, avec, ce qui introduit l'idée d'un lien de lutte avec un adversaire ou un partenaire, voire un ennemi... bref, d'un rapport nécessaire à l'autre. Il y a des conflits violents, de purs rapports de force abusive, comme il y a des conflits non violents, menant à la négociation, au compromis, à l'entente. Et conflit comme violence suppose une bonne dose d'agressivité.

La notion de pouvoir (poder) est un terme voisin et en quelque sorte englobant. Pouvoir, du latin potere, être capable de, avoir de la puissance, une force pour... Cette notion désigne ainsi la qualité des détenteurs de force et de puissance, physique ou symbolique, capables d'action sur les autres. Comme les autres notions connexes, le pouvoir est d'abord un terme général, qui peut signifier positivement l'accomplissement de celui qui exerce du pouvoir et contribuer aussi à celui de l'autre, au bien-être des autres. Dans ses formes extrêmes agressives, conflictuelles ou violentes, il prend plutôt le sens d'une domination ou du contrôle de l'autre, voire de sa destruction.

\section{PHÉNOMÉNOLOGIE DES FORMES DE LA VIOLENCE}

Dans les développements qui suivent nous nous inspirons du travail important de Jan Philipp Reemtsma dans son ouvrage Confiance et violence, 
Essai sur une configuration particulière de la modernité (REEMTSMA, [2008] 2011). Si nous tentons de cerner les caractéristiques essentielles, eidétiques, de la violence, elle apparaît d'abord dans l'utilisation de la force physique qui agit sur le corps des autres, sur leur être concret, matériel et psychique. Et nous pouvons distinguer trois formes de violence: localisante (délocalisante), raptive ou abusive et autotélique.

La violence localisante désigne une action contre les autres définis comme de simples obstacles dans la poursuite des intérêts, intentions, projets des porteurs de violence. L'autre dans ce contexte n'a pas de réalité propre à prendre en compte. Il s'agit de l'éliminer ou le détruire. C'est le cas d'un état de guerre où l'autre est l'ennemi à abattre pour conquérier un espace, un point stratégique. C'est alors un «pur rapport de forces». Si l'autre fuit ou s'éloigne, la violence cesse. L'attaquant peut dire: je ne sais pas qui je tue ou élimine, ça me fait obstacle, ça occupe un lieu à conquérir. L'autre est réduit à un être matériel, qui est là où il ne faut pas, qui doit être délocalisé. C'est aussi ce que l'on peut appeler une violence instrumentale, où l'autre est défni en fonction d'une visée supérieure et n'a aucune importance en soi. Cela peut inclure par exemple le meurtre sur commande qui fait de l'autre simplement «la cible» à abattre.

La violence raptive, captative, est une action contre l'autre, mais un autre dont cette fois on a besoin pour réaliser ses intérêts et ses désirs, qui a non seulement une réalité propre qui est bien reconnue, mais dont l'un a besoin pour satisfaire ses projets, de façon unilatérale. Raptive, cette violence repose sur une utilisation abusive des qualités de l'autre pour soi. Le cas du viol pour satisfaire un besoin sexuel est exemplaire. Mais cela peut s'étendre à tout le registre de l'activité humaine abusive. L'esclavage, le travail forcé ou contraint en constituent d'autres exemples, suivant un schéma d'interaction bien rendu par F. Hegel (1941), dans ce fameux rapport du Maître et de l'Esclave.

La violence autotélique est une forme extrême de violence raptive en quelque sorte, puisque c'est aussi une action qui procède d'un besoin de l'autre, mais cette fois-ci uniquement pour satisfaire son désir de puissance et de maîtrise totale de l'autre, jusqu'à sa destruction. Le cas type est celui de la torture qui exploite jusqu'à l'extrème la résistance d'autrui, du sujet vivant jusqu'à la mort. Autotélique, car il s'agit d'une violence, d'une force exercée pour elle-même et non pour autre chose.

Il faut souligner que la référence première de la violence est celle qui s'exerce sur le corps de l'autre, sa présence matérielle qu'il faut éliminer, utiliser ou dominer. Mais elle s'étend aussi à la violence que nous pourrions appeler psychologique ou symbolique qui s'adresse à l'autre comme sujet psychique et 
sujet social. Dans tous les cas, à des degrés divers, la violence est l'usage de la force ou la menace de son usage contre l'autre qui vise à réduire l'autre comme un objet, à le réifier, comme une chose dont on peut disposer. Suivant les diverses formes décrites ici, l'autre n'est rien (violence localisante), doit être soumis (violence captive), est dominé souverainement (violence autotélique).

\section{CONFIANCE ET VIOLENCE DANS LE LIEN SOCIAL, ÉMERGENCE DU POLITIQUE}

L'autre face des rapports humains, ce qui permet de vivre et de survivre ensemble c'est la construction d'une attitude de confiance, de pouvoir se fier, d'avoir foi en l'autre comme étant bienveillant à mon égard. Cette confianse se construit socialement sur la base d'une expérience antérieure avec les autres. Une relation de confiance n'enlève pas l'usage d'un pouvoir, d'une puissance exercée entre les personnes, mais ce pouvoir est vécu comme partagé et bénéfique pour chacun. Paradoxalement, la confiance repose sur une capacité réciproque, entre les être humains, d'agir «en puissance». Mais le rapport de confiance sur cette base peut facilement se transformer si l'autre s'impose, impose, abuse, bref exerce de la violence. Méfiance, défiance, perte de confiance et réponse de défense et de violence s'ensuivent. Comment sortir alors de ce rapport de force imprévisible, du règne de la «loi du plus fort»?

C'est ici qu'appararait l'importance de l'institution, de cet établissement par les êtres humains de règles et de normes autour de valeurs partagées, ne seraitce minimalement que pouvoir répondre à ses besoins fondamentaux et le projet pour chacun de bien vivre. L'institution de règles communes suppose un pouvoir médiateur, délégué, qui veille à leur applications pour le bien commun. C'est ici que naît le politique dans une société. Et sans refaire ici la longue histoire de l'humanité, il faut noter que ce triptyque «confiance, violence, pouvoir politique» est à penser au fondement même du vivre ensemble social du plus loin que l'on peut remonter dans les sociétés dites prémodernes. La naissance des groupes, des clans, de collectifs ethnoculturels, des cités et des pays est fondée sur cette dynamique avec des formes de pouvoir sans doute fondées sur la force physique, mais aussi sur des compétences et des marques symboliques, des références aux rituel et au sacré, et toute un ensemble de ressources matérielles et culturelles. Cette importance des institutions sociales nous conduit jusqu'à la modernité qui en constitue un moment fort.

La dynamique des rapports entre confiance et violence est en quelque sorte médiatisée par le pouvoir, par ceux qui détiennent les ressources utiles ou 
nécessaires pour que les autres puissent tous mieux vivre ensemble. Ce pouvoir plus ou moins centralisé est source possible de gratifications, de réponse positive aux besoins exprimés par les citoyens, ou au contraire, de sanctions sur toute conduite humaine perçue comme déviante. Une gratification qui se concrétise par des mesures ou avantages concrets pour la population est source d'une confiance dans le pouvoir et les institutions. Par contre, une violence sociale risque de se développer selon diverses voies. Une promesse de gratification qui n'est pas réalisée ou encore, un acquis social qui est arbitrairement réduit ou retiré, ou un ensemble de sanctions qui apparaissent injustes constituent les bases d'une rupture plus ou moins radicale de cette confiance institutionnelle et favorisent des conduites de violence et d'un retour aux purs rapports de force anomique, en deça des règles sociales du droit.

Une lecture intéressante pour saisir autrement la question de la confiance est celle d'Axel Honneth, dans ses deux ouvrages complémentaires, La lutte pour la reconnaissance (HONNETH, 2000) et la Société du mépris (HONNETH, 2006). Sont distingués trois types de reconnaissance qui sont autant de dimensions du lien social. Il y a d'abord la reconnaissance de l'être humain qui vaut comme être humain globalement, au sein des relations sociales primaires, d'abord la relation maternelle, puis celles de la famille, des amis, des amoureux qui sont le lieu privilégie d'accepter l'autre telle qu'il est, ce qui en retour favorise l'amour de soi. Paul Ricoeur (1990) parlera de cette condition existentielle fondamentale qu'est la relation de sollicitude à l'égard d'autrui, condition d'une confiance en l'autre et confiance en soi tout aussi radicale.

En même temps, dans le contexte de la société, c'est l'exercice d'une reconnaissance des droits et des devoirs de toute personne, fondée sur le traitement égalitaire et solidaire de tous les «citoyens», des membres de la Cité, sur des institutions justes. Cela permet le développement d'un sentiment de dignité, fondé sur le statut social qui permet la confiance en l'autre en tant que citoyen. Enfin, il existe une troisième forme de reconnaissance qui est liée à l'activité et à la compétence de chacun ou chacune dans la contribution qui est apportée au groupe social par son travail, ses réalisations, ses idées, sa créativité. Cette reconnaissance est celle des pairs et des autorités dans les divers contextes d'activité, dont en particulier les milieux de travail. Elle favorise l'affirmation réciprope de l'estime de soi, d'une confiance dans la valeur «productive» de l'autre.

Il est aisé de voir la contrepartie critique du développement possible d'une société du mépris qui vient miner les bases des ces formes de reconnaissances: éclatement des formes de relations primaires et de leur qualité, liées en partie aux politiques sociales insuffisantes; non respect récurrent des droits dans 
les diverses formes de discrimination sociale, créatrice d'inégalités sociales, ce que Ricoeur (1990) appelle les institutions injustes; et enfin, un monde du travail et une hiérarchisation des activités dominés par le seul critère de la performance rentable dans une économie de profits et de consommation, suivant l’idéologie néolibérale.

\section{VIOLENCE ET MODERNITÉ EN PERSPECTIVE}

Le projet de la modernité, d'histoire relativement récente, comporte une dimension régulatrice de ce qui pouvait encore persiste de l'arbitraire de la force et de la violence dans les divisons sociales et dans les formes du pouvoir souverain, fondé sur l'appartenance à un clan dominant, à la transmission héréditaire des chefs et des rois et d'une sacralisation du pouvoir dans les sociétés dites prémodernes. La modernité présente une construction sociale qui serait fondé sur la connaissance et la science (les Lumières de l'esprit) appelé à remplacer les forces dominatrices d'une transcendance religieuse, sur la liberté de penser et d'agir des individus dans un cadre démocratique (le pouvoir du peuple, par le peuple, pour le peuple), d'une économie fondée sur le travail et l'organisation industrielle que permet le développment de la science et des technologies, tout cela dans une idéologie du progrès que vont symboliser des concepts fondateurs de la révolution française: liberté, égalité, fraternité. Ce modèle d'origine occidentale offrirait alors une garantie fondamentale d'un vivre ensemble fondé sur une société des droits humains qui prendrait la forme d'États nationaux démocratiques où un régime de droit vise à contenire toute violence abusive, l'interdire, la sanctionner sévèrement. Pour ce faire cependant, il faut maintenir une violence «légitime», avec la police, l'armée, l'appareil judiciaire. L'État doit pouvoir se défendre contre les attaques possibles de l'extérieur, par d'autres États, où, de l'intérieur, contre les conduites de toute personne ou groupe qui, par des actes de violence, ne respecte pas les lois et les institutions. Les représentants de l'État peuvent alors exercer une forme de violence légitime, localisante, «la guerre juste», le retrait pénitentiaire ou autre sanction jugées nécessaires.

J. P. Reemstma fait par ailleurs une analyse historique critique de ce qu'il appelle la relégitimaition de la violence dans la modernité, montrant, par des exemples puisés dans de grands événements du XXième siècle comment la violence légitime, surtout délocalisante et instrumentale, prête flanc et peut régresser, en toute légitimité officielle, à la violence raptive ou autotélique. L'exemple du régime nazi et de la solution finale dans la volonté d'extermination des juifs l'illustre fortement. Au nom d'une vision étatique nationaliste et raciste, 
le travail forcé, la torture et l'extermination des Juifs comme ceux de groupes marginalisés (personnes déficientes, prisonniers politiques, romanichels etc.) sont légitimés par le pouvoir conquérant. Des expérimentations nucléaires, celle des États-Unis au Japon, où diverses formes de torture fréquentes dans plusieurs pays en guerre, ou en lutte contre le terrorisme en sont d'autres exemples. La fréquence récurrente de meurtres, de viols, de diverses formes d'exploitation phsyique ou symbolique dans nos pays contemporains témoignent aussi des limites de ce pouvoir légitime qui ne réusssit pas à contrer ces formes excessives de violences et qui, à son tour, autorise au nom de son pouvoir légitime des violences abusives.

\section{LES LIMITES DU CONTRAT SOCIAL: HOBBES, ROUSSEAU, FREUD}

D’où vient la violence? Est-ce une disposition innée chez l'humain, une pulsion radicale comme l'évoque Freud dans la notion de pulsion de mort ? Où une apparition historique liée à des rapports de domination des forts contre les faibles, de la société contre l'individu ? On reconnaît là à la fois l'opposition entre les thèses philosophiques d'un auteur comme Thomas Hobbes, Léviathan (HOBBES, [1651] 1991) qui étudie les fondements de l'État et de la souveraineté, le contrat social qui est à la base du Pouvoir souverain est présenté comme une institution nécessaire contre la violence radicale de l'homme « naturel », dont la liberté individuelle est source d'excès et de violence pour autrui, «l'homme est un loup pour l'homme» (de Plaute: Homo homini lupus). Rousseau, dans le Contrat social (ROUSSEAU, [1762] 1963), arrive aussi à la nécessité d'établir un Contrat social, mais pour contrer cette fois l'arbitraire d'une Société qui «corromp»» l'individu naturel, dont l'orientation spontanée est de bonté et d'harmonie. Freud, pour sa part, montre la nécessité du processus de civilisation pour contrer ou du moins réduire les formes radicales des violences liées à la pulsion de mort. La psychologie humaniste d'un Carl Rogers (1968), aux antipodes de Freud et souvent rapprochée des thèses de Rousseau, montrant comment la société est source des problèmes et de la violence, se dressant contre un individu dont l'orientation fondamentale est positive, ouverte à la socialité et aux liens constructifs avec autrui.

Freud, dans ses œuvres dites «sociales», en particulier Totem ettabou (FREUD, 1973), tente de fonder le lien social sur une violence originelle de l'humanité et ce à partir de références historiques et anthropologiques. En effet, le meurtre du Père et l'interdit de l'inceste, tels que puisés aussi bien dans les règles d'exogamie clanique que dans sa lecture de la tragédie «Oedipienne», le conduisent à fonder 
le complexe d'CEdipe marquant les rapports enfants et parents et les interdits associés. Plus largement encore, le récit inspiré de certains travaux ethnologiques sur le meurtre collectif du Père de la Horde serait fondateur de l'institution du lien social entre les «frères».

La réflexion d'Eugène Enriquez, dans De la Horde et l'État (ENRIQUEZ, 1983), reprend aussi ce questionnement des sources de la violence, s'appuyant sur Freud, mais éclairé par les travaux anthropologiques plus récents et des travaux de sociologie politique. Il partage l'hypothèse d'une violence radicale d'origine sociale et psychique, montrant comment Freud dépassait dans ses œuvres dites sociales la seule préoccupation individuelle et psychique. Par contre, il demeure plus proche de la thèse d'un champ pulsionnel partagé, chez l'homme, entre pulsion de vie et pulsion de mort, double direction pulsionnelle qui en quelque sorte fait que ces deux pulsions sont inséparables : libido, sexualité et tendance destructrice et mortifère. Référant à Freud (1973) qui dans Totem et tabou, établit une violence primitive du parricide collectif, fondateur de la communauté des frères, il montre que c'est la transgression de toute transgression, c'est celle qui vise l'autorité du chef, du Père. Il devient alors nécessaire pour garantir le lien social de dresser des interdits du parricide et de l'inceste, mais cette base normative est toujours menacée par le retour de la violence transgressive et du chaos.

Par ailleurs, Enriquez (1983) insiste sur l'importance des forces d'attraction, de la pulsion libidinale, de la poursuite de l'amour comme également fondatrices du lien social. Il y a solidarité et liens amoureux possibles, pulsion de vie, désir et sexualité, créativité et langage. L'auteur montre, sur cette base, qu'il y a effort constant dans l'évolution des sociétés, de marquer la différence et l'altérité, pour créer de l'ordre, de l'institution, des règles gouvernant les rapports sociaux. Mais là encore réapparaît, au sein même de ce travail de classification, d'ordonnancement des rapports sociaux, un processus récurrent de domination, expression instituée de la violence. Ces rapports de dominations suivent d'abord des distinctions anciennes dont il fait ensuite l'examen: l'ordre des sexes, où le masculin tente de dominer le féminin (thèse du patriarcat); l'ordre et le conflit des générations; la hiérarchie nature et culture, où l'homme devient progressivement «maître» et exploiteur de la nature; l'ordre cosmologique et les religions, où l'homme est tantôt soumis au divin ou au surhomme. Ce sont autant de bases fondamentales des distinctions, sources de régulation qui, perverties, sont investies de rapports de domination. Un tel travail de domination se poursuit dans les institutions des sociétés modernes avancées, dont les modes de gouvernance régnant dans les États et le monde de l'économie. Une attention particulière est alors accordée au 
cas historique majeur de l'antisémitisme nazi, figure d'excès du pouvoir souverain de l'État national fondé sur l'identité ethnique.

Il y aurait ainsi, toujours, une dialectique radicale des pulsions, pulsion de vie et pulsion de mort, amour, sexualité, et violence. Prenant en compte le contexte contemporain de nos sociétés, poursuivons notre questionnement sur cette difficulté radicale de vivre ensemble en reprenant la question de la modernité.

\section{LA CRISE DE LA MODERNITÉ ET SES FIGURES VARIÉES}

Que se passe-t-il depuis une cinquantaine d'années qui nous fait penser effectivement au dépassement ou à l'échec du grand projet de la modernité? Où sommes-nous simplement devant une autre crise, éphémère, un redéploiement des forces sociales, une nouvelle étape sur la voie de la modernité? Il semblerait que les changements soient plus radicaux. Seconde modernité, modernité avancée, postmodernité, hypermodernité, troisième industrialisation sont autant de notions voulant signifier des changements de grande ampleur. C'est aussi une vision radicalement différente de l'individu sujet et acteur social qui prendrait place. Et la problématique du pouvoir, de la violence, de la confiance sont à resituer au sein de cette crise.

\subsection{UNE PHASE POSTMODERNE ET LA FIGURE D'UN NOUVEAU SUJET ROMANTIQUE}

Quand nous lisons certains écrits fortement inspirés du postmodernisme, de Jean-François Lyotard (1979) à Jean Baudrillard (1970), de Zygmunt Bauman (2007) à Gilles Lipovetsky et Sébastien Charles (2004) ou Michel Maffesoli (1988), nous oscillons entre une vision tragique à la Nietzsche du Surhomme destiné à remplacer Dieu dont la mort est déclarée ou une vision romantique de l'individu enfin débarrassé des contraintes des grands récits modernes ou de ses idéaux normatifs jugés idéalistes et hypocrites. Le progrès vers une société égalitaire et juste, la solution scientifique aux grands problèmes de la nature ou de la société, de la croissance et de la richesse, voire tout grand récit sont illusoires. Ce qui est en cause dans le discours postmoderne, c'est l'ébranlement des grandes institutions de la modernité. C'est la remise en cause de la souveraineté politique fondée sur des règles et des valeurs morales pour tous, la remise en cause des fondements mêmes de la raison, source de règles universelles fondées sur la science qui s'impose comme norme de la connaissance et de la 
vérité. Finalement, c'est la critique du faux espoir, de l'utopie d'une implication solidaire des humains dans la visée d'un progrès matériel et symbolique d'une société juste et démocratique à venir. Le discours postmoderne insiste sur les limites et les échecs de la science, de la morale, de l'État, des grandes utopies politiques devant la complexité des problèmes sociaux et fait appel aux seuls choix et aux seules responsabilités individuelles, à la liberté, aux individus sujets et créateurs de leur mode de vie, dans le présent. Ce mouvement de pensée se développe d'abord dans le milieu artistique de l'après-guerre et trouve en partie son expression dans les diverses manifestations contestataires des années 19601970 sous le sceau de la «contre-culture».

Cette phase postmoderne trouve son écho dans cette montée, à la fin du $\mathrm{XX}^{\mathrm{e}}$ siècle, dans la construction sociale d'une culture narcissique comme idéologie dominante (LASCH, 2000) et un individualisme croissant, résultat de la grande fragmentation du social et des institutions. Ces manifestations expriment la quête d'un sujet romantique autonome, créateur de sa vie propre, à distance de toute institution hétéronome. Le politique étatique et national, le progrès scientifique ou économique, le monde de la raison scientifique sont autant de dimensions devenues arbitraires et lointaines face à cette affirmation d'un sujet désirant et indépendant. Mais ce que nous retenons surtout, c'est la méfiance radicale à l'égard de toute institution universalisante issue du projet de la modernité.

\subsection{L'HYPERMODERNITE NEOLIBERALE ET LA FIGURE D'UN SUJET LIBREMENT ASSUJE'T'TI}

Une relecture critique des tenants de la postmodernité, parfois réalisée par eux-mêmes, et d'autres auteurs comme Sébastien Charles (2007), Gilles Lipovetsky et Sébastien Charles (2004), ou autrement, Bruno Latour (1997), Nicole Aubert (2004), Vincent de Gaulejac (2005), pointe dans une autre direction, non moins troublante: le passage progressif vers une hypermodernité, définie comme l'excès de la modernité. La libération de l'individu des contraintes institutionnelles conduit, selon cette perspective, à un individualisme radical, rendant en cela même très limité l'exercice de sa liberté; la valeur de la connaissance scientifique est encore reconnue, mais seulement quand elle devient appliquée et technique, «utile»; la gouvernance démocratique est encore invoquée, mais devient simplement l'exercice supérieur du management des affaires publiques; enfin, la productivité n'est plus dirigée vers un progrès lointain de l'humanité, mais vers la consommation et le profit. 
La logique moderniste n'est pas niée: elle est développée à l'excès comme imposition d'une La nouvelle raison du monde (DARDOT; LAVAL, 2010), dominante et d'un individualisme exacerbé. C'est l'homme unidimensionnel de Marcuse (1968), abrité et soumis à la fois au règne de la pensée instrumentale, ce nouveau Grand récit d'un pragmatisme radical, pourrions nous dire. C'est une nouvelle modernité qui glorifie le présent, l’intensité, le performatif, sans le poids du passé, ni l'exigence du futur. Une pensée opérationnaliste radicale se meut au rythme de la consommation des besoins et de la performance de l'action, privilégiant le court terme, au mieux, le moyen terme si des obstacles évidents se posent. Il y a eu sans doute un moment postmoderne dans plusieurs secteurs de nos sociétés, permettant d'affaiblir un peu plus l'imposition des normes universelles ou des grands projets émancipatoires du futur (TAYLOR, 2007). Ce moment postmoderne aurait ainsi accéléré le passage à l'hypermodernité.

Reprenons une définition synthétique des quatre pôles de la modernité confrontée à une lecture hypermoderne, nous inspirant en particulier de l'ouvrage de Dardot et Laval (2010). L'économie néolibérale devient dominante, commandant un État gestionnaire «fort» mais limité au mandat principal d'assurer le développement des libertés individuelles seules nécessaires à un marché libre. Le monde du travail et de l'entreprise est soumis à des impératifs de performance et de concurrence incessants, dans une perspective de mondialisation, soutenu en cela par des progrès technologiques majeurs, ceux de l'informatique et des réseaux de communication, caractéristiques d'une troisième grande phase industrielle ${ }^{1}$. Une culture de consommation domine, sans l'espoir d'un progrès futur quelconque, mais plutôt axée sur un idéal d’intensité et de jouissance immédiate, du maintenant. L'individu libre et responsable est alors une figure centrale, seul pour conduire sa vie et faire ses choix; c'est «l'individu-entrepreneur», appelé à choisir sa vie dans toutes ses dimensions pour se réaliser.

L'individu dans ce contexte d'hypermodernité est de plus en plus mesuré à l'aulne de sa valeur productive, dans la sphère du travail, de la vie privée, de la consommation. Il devient l'élément essentiel d'une économie et d'une gouvernance néolibérales des sociétés marquées par l’idéologie hypermoderne. $\mathrm{Au}$ modèle moderne classique où le politique (la démocratie) dirigeait la production et le travail qui commandait l'économie de marché, nous avons maintenant une tendance inversée: l'économie commande la production et la politique. Et la citoyenneté est ébranlée dans sa base, mesurée par sa

La première révolution industrielle ou première industrialisation se situerait dans la période 1780-1850, avec la machine à vapeur, l'usine, le charbon... La seconde industrialisation (18501950) connait la grande industrie, les nouvelles formes d'énergie comme l'électricité, des machines de plus en plus automatisées. 
rentabilité économique et finalement, sociale. Ce néo-productivisme introduit une nouvelle échelle de rapports sociaux: au haut de l'échelle, des individus et des organisations performantes, très valorisées; une classe moyenne réduite, représentant des réalisations jugées plus traditionnelles, nécessaires mais dévalorisées; des groupes de plus en plus importants de marginalisés et d'exclus. L'idéal démocratique au sens fort est devenue un monde oligarchique où la règle d'or est la performance et la richesse.

Il faut nuancer fortement un tel portrait aussi schématique, quand nous prenons en compte toute l'immense diversité historique et culturelle des sociétés, non seulement occidentales mais celles de l'Amérique du Sud, de l'Afrique, de l'Asie. Et nos États-Nations sont marqués par une diversité interne aussi très forte: diversité ethnoculturelles, milieux urbains et paysans, inégalités sociales et groupes marginalisés. Il s'avère par ailleurs que le contexte actuel des échanges économiques, sociaux et politiques internationaux donne une impulsion considérable à la thèse de l'hypermodernité néolibérale et néoproductiviste en particulier, par le délitement des interactions fortes, constitutives du modèle industriel et sociétal dominant le $\mathrm{XX}^{\mathrm{e}}$ siècle. En effet, la mondialisation des échanges et surtout, la globalisation de ces échanges autour d'un primat hégémonique des intérêts d'un néocapitalisme financier ébranlent les liens sociaux définis au sein des sociétés nationales. La dissociation relative du capital, du travail productif et du marché et de la consommation en est la marque effective. La crise de confiance montante à l'égard du politique et l'affaiblissement des mouvements sociaux sont d'autres signes fréquents liés à ladite crise des Étatsnations et surtout de l'État-providence, ce régulateur socialisant. Nous pourrions tout autant énumérer toute une série de remises en cause collatérales touchant les règles morales et l'éthique, les institutions comme la famille, la valeur du travail, l'école, etc. Que voilà terrain propice à l'expression renforcée de la montée des violences «hypermodernes».

\section{CONCLUSION. QUE FAIRE?}

La dialectique profonde entre la confiance et la violence dans la construction du lien social repose sur des rapports de pouvoir et ultimement sur la qualité démocratique d'une société, comme on a pu le définir dans le projet de la modernité. Notre brève incursion dans les formes actuelles qui marquent la crise de la modernité nous permet de mettre en évidence celle de la démocratie. Le pouvoir du peuple est en fait, à des degrés divers, le pouvoir des plus forts sur le peuple exerçant au nom de la volonté commune les intérêts 
d'une partie dominante. Qui dit force dominante, dit aussi groupes dominés et rapport de forces où les sanctions s'exercent, les déceptions face l'injustice, futelle légale exarcébées. Une perte de confiance s'ensuit vis-à-vis les institutions, pour beaucoup, et peut conduire à diverses formes de violence instituée, et, en contrepartire, à des violences arbitraires de rébellion, de survie, d'affirmation dans la marginalité.

Notre contribution comme chercheur ou professionnel en sciences humaines et sociale est sans doute fort limitée. Mais il reste à faire et refaire constamment oeuvre critique dans la compréhension des rapports entre violence et politique dans notre société. Critique, il faut confronter les diverses interprétations théoriques de cette thématique et élargir à d'autres interprétations. La complextié du réel d'une société ne peut se réduire à une lecture globalisante comme celle des conceptions de la modernité ou de ses crises. Une pensée critique se doit de relier ces interprétations comme autant d'hypothèses à valider par des études plus empiriques. Comment cela se passe-t-il effectivement dans le monde du travail, en éducation, dans la famille, dans les arts, les pratiques religieuses, en ville, dans les campagnes? Et dans tel pays, telle ville ou région, dans période temporelle?

S'il faut documenter les tendances politiques et idéologiques créatrices d'inégalités sociales, de politiques de «mépris» à l'égard de larges segments de la population, il est nécessaire aussi de montrer celles qui continuent à s’inspirer du projet moderne. S’il faut repérer les formes de violences instituées et les violences réactives ou désespérées, sur fond d'une perte radicale de confiance dans les institutions et au pouvoir politique institué, mis en place, il faut aussi reconnaître les nouvelles formes de résistance et d'action sociale, les luttes pour la reconnaissance, les solidarités locales et associatives, la valeur accordée au travail professionnel, au métier, l'investissement dans les relations primaires et les familles. Comme chercheur et intervenant, c'est de jouer ce rôle nécessaire de médiation entre ces forces positives de résistances et ces forces destructives de violence.

En ce sens, nous inspirant de nos propres travaux de recherche et d'intervention (RHÉAUME, 2017; FRITZ; RHÉAUME, 2014), nous accordons une grande importance que a ce que nous pouvons qualifier d'approche clinique en sociologie et en sciences sociales. Cette approche clinique s'appuie sur une forme de recherche-action participative qui se fait avec des partenaires sociaux de milieux communautaires ou associatifs, organisationnels, d'institutions publiques. Cette façon de faire de la recherche repose sur ce que Paulo Freire (1982) définissait déjà comme approche dialoguale, un échange de savoirs entre la connaissance scientifique des chercheurs, le savoir d'expérience et le savoir 
professionnel des autres participants. Une production de connaissance qui reconnait la valeur propre de chacune de ces formes de savoirs et qui permet par des échanges et la confrontation, le développement spécifique de ces savoirs différents. Un travail de recherche et d'intervention qui consiste à reconstruire du lien social et de confiance dans un mouvement mobilisateur des collectifs instituants, pour reprendre cette notion de Cornelius Castoriadis (1975), qui, malgré le poids de l'institué créateur d'inégalité et de domination du modèle dominant actuel d'une démocratie représentative d'une minorité, rappelle que tout institué est produit par des forces instituantes, par le peuple dans son entier et que le projet démocratique pour tous est politiquement possible.

\section{REFERENCES}

AUBERT, N. (org.). L'individu hypermoderne. Paris: Éditions Érès, 2004.

BAUDRILLARD, J. A sociedade de consumo. Lisboa: Edições 70, 1970.

BAUMAN, Z. Liquid modernity. Cambridge: Polity Press, 2005.

CASTORIADIS, C. L'institution imaginaire de la société. Paris: Éditions du Seuil, 1975.

CHARLES, S. L'hypermoderne expliqué aux enfants. Montréal: Liber, 2007. DARDOT, P.; LAVAL, C. La nouvelle raison du monde: essai sur la société néolibérale. Paris: La Découverte, 2010.

ENRIQUEZ, E. De la horde à l'État. Essai de psychanalyse du lien social. Paris: NRF, Éditions Gallimard, 1983.

FREIRE, P. Pédagogie des opprimés, suivi de Conscientisation et révolution. Paris: Librairie François Maspero, 1982.

FREUD, S. Totem et Tabou. Paris: Payot, 1973.

FRITZ, J. M.; RHÉAUME, J. (ed.). Community intervention. Clinical sociology perspectives. New York: Springer, 2014.

GAULEJAC, V. de. La société malade de la gestion: idéologie gestionnaire, pouvoir managérial et harcèlement social. Paris: Seuil, 2005.

HEGEL, F. La phénoménologie de l'esprit. Traduction Jean Hyppolite. Paris: Aubier, Montaigne, 1941.

HOBBES, T. Leviathan. Editor: R. Tuck Cambridge. Tradução: F. Tricaud. Paris: Sirey, [1651] 1991. 
HONNETH, A. La lutte pour la reconnaissance. Paris: Les Éditions du CERF, 2000.

HONNETH, A. La société du mépris. Vers une nouvelle théorie critique. Paris: La Découverte, 2006.

JOHNSON, R. N. Agression in man and animals. Philadelphia, London, Toronto: Sauders Editor, 1972.

LASCH, C. La culture du narcissisme. La vie américaine à un âge de déclin des espérances. Paris: Climats, 2000.

LATOUR, B. Nous n'avons jamais été modernes: essai d'anthropologie symétrique. Paris: La Découverte, 1997.

LIPOVETSKY, G.; CHARLES, S. Les temps hypermodernes. Paris: Grasset et Fasquelle, 2004.

LYOTARD, J-F. La condition postmoderne. Paris: Éditions de Minuit, 1979.

MAFFESOLI, M. Le temps des tribus: le déclin de l'individualisme dans les sociétés de masse. Paris: Méridiens Klincksieck, 1988.

MARCUSE, H. L'homme unidimensionnel. Essai sur l'idéologie de la société industrielle avancée. Paris: Éditions de Minuit, 1968.

REEMTSMA, J. P. Confiance et violence. Essai sur une configuration particulière de la modernité. Paris: NRF, Gallimard, [2008] 2011.

RHÉAUME, J. Subject and hypermodernity. Psicologia, Conocimiento y Sociedad, Montevideo, v. 6, n. 2, p. 223-241, 2017. Disponível em: https: / / revista. psico.edu.uy/index.php/revpsicologia/issue/view/49. Acesso em: 02 maio 2017.

RICOEUR, P. Soi-même comme un autre. Paris: Éditions du Seuil, 1990.

ROGERS, C. Le développement de la personne. Paris: DUNOD, 1968.

ROUSSEAU, J-J. Du contrat social. Paris: Union Générale d'Éditions, [1762] 1963. (coll. 10/18, 89-90).

TAYLOR, C. Grandeur et misère de la modernité. Montréal: Bellarmin, 2007. 